\title{
The Sensitivity of TE Filed Propagating in LHM-Antiferromagnetic Sensor
}

\author{
Zeyad I. Al-Sahhar ${ }^{1}$, Hala J. El-Khozondar ${ }^{2 *}$ and Mohamad M. Shabat ${ }^{3}$ \\ 1. Physics Department, Al-Aqsa University, Gaza P. O. Box 4051, Palestine \\ 2. Electrical Engineering Department, Islamic University, Gaza P. O. Box 108, Palestine \\ 3. Physics Department, Islamic University, Gaza P. O. Box 108, Palestine
}

Received: February 20, 2014 / Accepted: March 08, 2014 / Published: April 25, 2014.

\begin{abstract}
A three-layer waveguide structure sensor consists of LHMs (left-handed materials) film surrounded by dielectric cladding and antiferromagnetic substrate is proposed. LHMs known as MTMs (metamaterials) have simultaneous negative permeability and permittivity. The dispersion relation for the structure is derived for TE guided modes. Two ranges of frequencies are chosen such that Voigt permeability, $\mu_{v}$, either negative or positive. The sensitivity is proven to be affected by different parameters including the film thickness, LHM parameters, and Voigt frequency.
\end{abstract}

Key words: MTMs, LHMs, antiferomagntic, optical waveguide sensors, sensitivity.

\section{Introduction}

A sensor in general recognizes and senses the presence of an analyte (the specie to be detected). The ideal sensor not only has to respond to low concentrations of analytes, but also must have the ability to discriminate among species. Sensors have a broad range of applications in medical, pharmaceutical, environmental, defense, bioprocessing or food technology.

The need for fast, easy to use, compact and cheap sensors is the motivation of this study. In this paper, the proposed structure is a three-layer system consists of new man-made materials named metamaterials as film sandwiched between an antiferromagnetic substrate and dielectric cladding. Antiferromagnetic metal is studied extensively because its negative dielectric constant influences the dispersion relation in a qualitative manner.

Ritchie [1] first study led to the fast growing field

\footnotetext{
*Corresponding author: Hala J. El-Khozondar, Professor, research field: electrooptics. E-mail: hkhozondar@iugaza.edu.ps.
}

of plasmonics owing to their potential application in areas as diverse as biosensing [2]. Several studies is conducted to understand both surface magnetic polaritron (plasmons) and guided waves in magnetic (anti/ferromagnetic) layered waveguides [3-10] where magnetic layered intercalated with dielectric layers or MTMs (metamaterials) layers.

LHM (left-handed materials) or MTMs are materials, which have been fabricated in the laboratory. Veselago [11] theoretically predicated the existence of LHM with both negative permeability ( $\mu$ $<0)$ and negative permittivity $(\varepsilon<0)$ and verified experimentally by Pendry et al. $[12,13]$ and Shelby et al. [14]. These materials have unique electromagnetic properties made them attractive to several applications including antenna design, superlenses, higher resolution magnetic resonance imaging [15, 16], sensors [17-19] and isolators [20,21].

The dispersion relation for the proposed structured is derived for TE (transverse electric fields) propagating modes only. The homogenous sensitivity, $S$, is defined as the change in the effective refractive 
index with cladding index [22], is then calculated to measure the ability of the proposed structure to detect and sense small changes at the cladding. Next section is devoted to cover the background. Numerical calculations and result discussions are given in Section 3. The paper is concluded in the Section 4.

\section{Theoretical Background}

Fig. 1 displays the three-layered waveguide sensor. The guiding layer consists of LHM sandwiched between a semi-infinite linear dielectric cladding and a semi-infinite AFM (antiferromagnetic) substrate layer. While the LHM film has both negative permittivity $\varepsilon_{3}$ and negative permeability $\mu_{3}$, the linear dielectric cladding has positive permittivity $\varepsilon_{1}$ and positive permeability $\mu_{l}$. The AFM substrate has positive permittivity $\varepsilon_{2}$ and tensor permeability $\mu_{2}$ defined by Eq. (1).

$$
\mu_{2}(\omega)=\mu_{02}\left(\begin{array}{ccc}
\mu_{11} & j \mu_{12} & 0 \\
-j \mu_{12} & \mu_{11} & 0 \\
0 & 0 & \mu_{z}
\end{array}\right)
$$

where,

$$
\begin{array}{r}
\mu_{11}=1+\frac{2 \omega_{a} \omega_{m}\left(\omega_{1}^{2}-\omega_{02}^{2}-\omega^{2}\right)}{\Delta} \\
\mu_{12}=\frac{4 \omega_{a} \omega_{m} \omega_{02} \omega}{\Delta} \\
\Delta=\left[\omega_{1}^{2}-\left(\omega+\omega_{02}\right)^{2}\right]\left[\omega_{1}^{2}-\left(\omega-\omega_{02}\right)^{2}\right] \\
\omega_{1}=\gamma_{2} \sqrt{H_{a}\left(H_{a}+2 H_{e}\right)}
\end{array}
$$

$\omega_{02}=\gamma_{2} H_{0}, \omega_{a}=\gamma_{2} M_{a}, \omega_{m}=4 \pi \gamma_{2} M_{s 2}, \omega$ is the circular frequency, $H_{0}$ is the external magnetic field that is applied in the $z$ direction and perpendicular to the direction of propagation of the waves, $M_{s 2}$ is the unscreened saturation magnetization in the direction of $H_{0}, \gamma_{2}$ is the absolute value of the gyromagnetic factor in the antiferromagnet, $H_{a}$ is the internal anisotropy field confined in the $x$ direction, and $H_{e}$ is the exchange field.

The bulk polaritons for antiferromagnet in an external magnetic have two frequency gaps [23, 24]. The two gaps correspond to the regions of negative value of the Voigt permeability:

$$
\mu_{v}=\left(\mu_{11}^{2}-\mu_{12}^{2}\right) / \mu_{11}
$$

where, $\mu_{\nu}$ has two poles at $\omega^{1}$ and $\omega^{3}$ and two zeros at $\omega^{2}$ and $\omega^{4}$ given by:

$$
\omega^{(1,3) 2}=\omega_{1}^{2}+\omega_{02}^{2}+\omega_{m} \omega_{a} \mp\left(4 \omega_{02}^{2} \omega_{1}^{2}+4 \omega_{m} \omega_{a} \omega_{02}^{2}+\left(\omega_{m} \omega_{a}\right)^{2}\right)^{1 / 2}
$$

$$
\omega^{(2,4)}=\mp \omega_{02}+\left(\omega_{1}^{2}+2 \omega_{m} \omega_{a}\right)^{1 / 2}
$$

The surface polaritons for the semi-infinite antiferromagnet in the Voigt configuration have two branches for each direction of propagation. The transverse fields TE are assumed to have the oscillation form as follows:

$$
\begin{array}{r}
\vec{E}=\left(\begin{array}{lll}
0 & 0 & E_{z}
\end{array}\right) e^{j(\omega t-k y)} \\
\vec{H}=\left(\begin{array}{lll}
H_{x} & H_{y} & 0
\end{array}\right) e^{j(\omega t-k y)}
\end{array}
$$

where, $k=N \omega / c$ is the propagation constant, $N$ is the effective refractive index, and $c$ is the speed of light. Applying Eqs. (9) and (10) to Maxwell's equations and applying boundary conditions at $x=$ $-a / 2$ and $x=a / 2$, we obtain the dispersion equation as follows:

$$
i F_{3}\left(F_{2}-F_{1}\right) \cot (\beta a)-\left(F_{1} F_{2}-F_{3}^{2}\right)=0
$$

where, $F_{1}=-j \lambda_{1}, F_{2}=-j \mu_{02}\left[\mu_{12}-\lambda_{2} \mu_{11}\right], F_{3}=j \lambda_{3}, \lambda_{1}=$ $N / \alpha_{1}, \quad \alpha_{1}=\left(N^{2}-n_{1}^{2}\right)^{1 / 2}, n_{1}=\left(\varepsilon_{1}\right)^{1 / 2}, \lambda_{2}=\left[N \mu_{11}-\right.$ $\left.\alpha_{2} \mu_{12}\right] /\left[N \mu_{12}-\alpha_{2} \mu_{11}\right], \alpha_{2}=\left(N^{2}-\varepsilon_{2} \mu_{v}\right)^{1 / 2}, \mu_{v}=\left(\mu_{11}^{2}-\right.$ $\left.\mu_{12}{ }^{2}\right) / \mu_{11}, \lambda_{3}=N \mu_{3} / \alpha_{3}, \alpha_{3}=\left(N^{2}-\varepsilon_{3} \mu_{3}\right)^{1 / 2}$, and $\beta=j \alpha_{3}$.

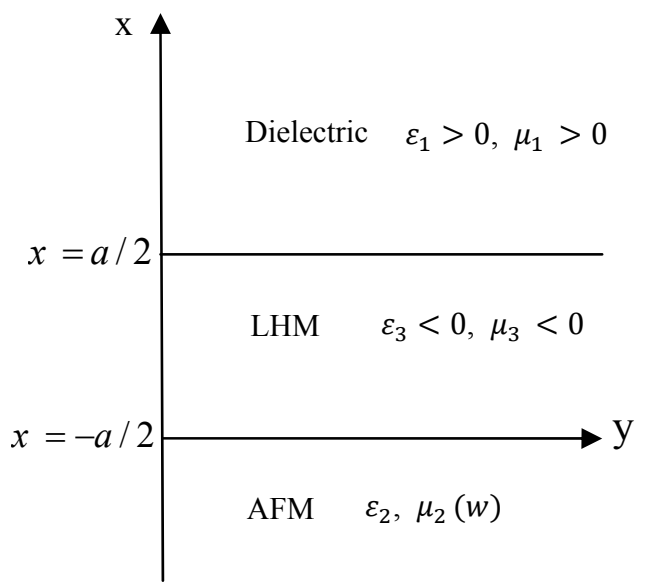

Fig. 1 The proposed three layered waveguide. The film is LHM with $\varepsilon_{3}<0, \mu_{3}<0$ surrounded by AFM substrate and dielectric cladding. 
The homogenous sensitivity due to guided waves, $S$, is calculated from the dispersion Eq. (11) by differentiating the effective refractive index $N$ with respect to the cladding refractive $n_{l}=\varepsilon_{l}^{1 / 2}$. So, $S$, can be expressed as Eq. (12).

$$
S(\omega)=\frac{\partial N}{\partial n_{1}}
$$

The calculation of $S$ is tedious; hence, it is not reproduced here. The values of the refractive index $N$ for surface waves are first numerically calculated form Eq. (11) and accordingly the sensitivities $S$ is calculated numerically from Eq. (12). The behavior of our system will depend on the values of $\omega^{1}, \omega^{3}, \omega^{2}$,

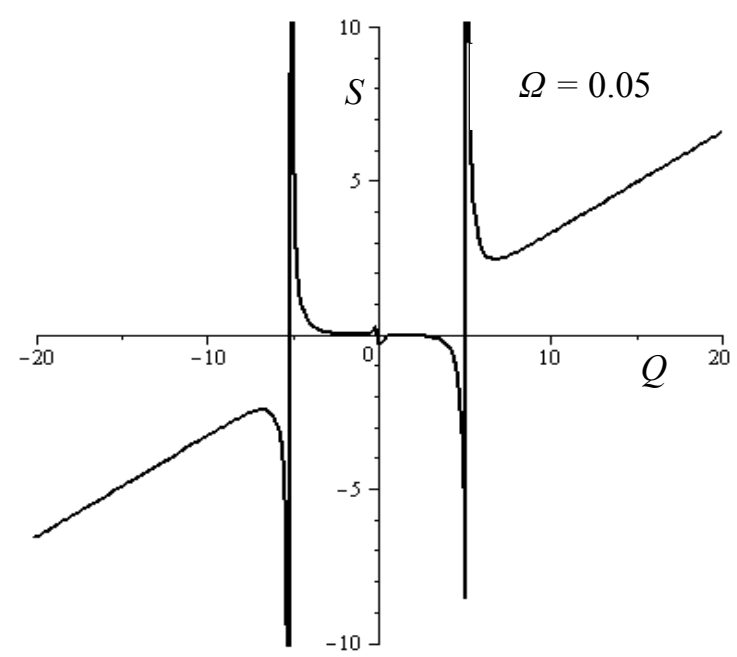

(a)

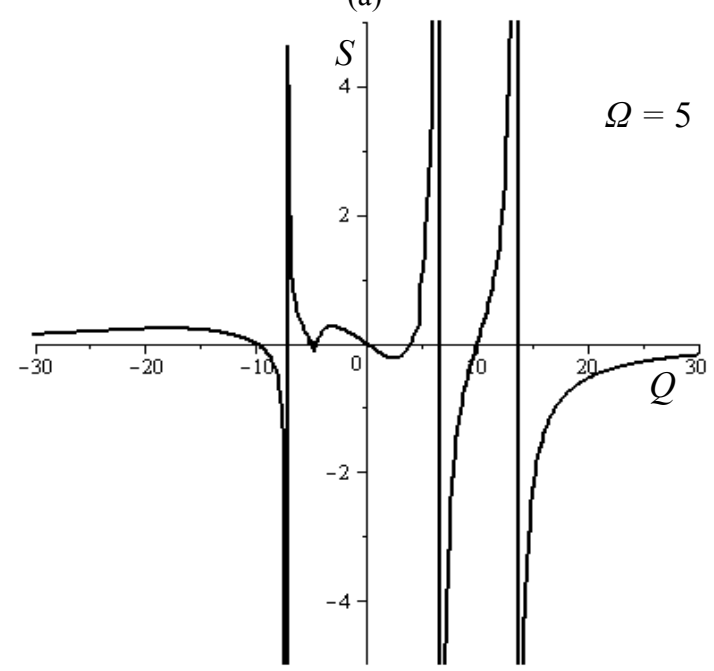

(c) and $\omega^{4}$. Moreover, it will be affected by the values of MTM's parameters and the thickness of the film.

\section{Results and Discussion}

In this work, we studied the sensitivity of the sensor. Moreover, we test the effect on the sensitivity for $\mu_{\mathrm{v}}<$ 0 and $\mu_{v}>0$. In the numerical calculation, we defined the reduced frequencies, $\Omega=\omega / \omega_{m}, \Omega_{0}=\omega_{02} / \omega_{1}, \Omega_{a}=$ $\omega_{a} / \omega_{1}, \Omega_{m}=\omega_{m} / \omega_{1}$ and wave number, $\mathrm{Q}=k c / \omega_{m}=$ $N \Omega$.

The sensitivity is calculated for both cases when $\mu_{v}$ $<0$ and $\mu_{v}>0$.

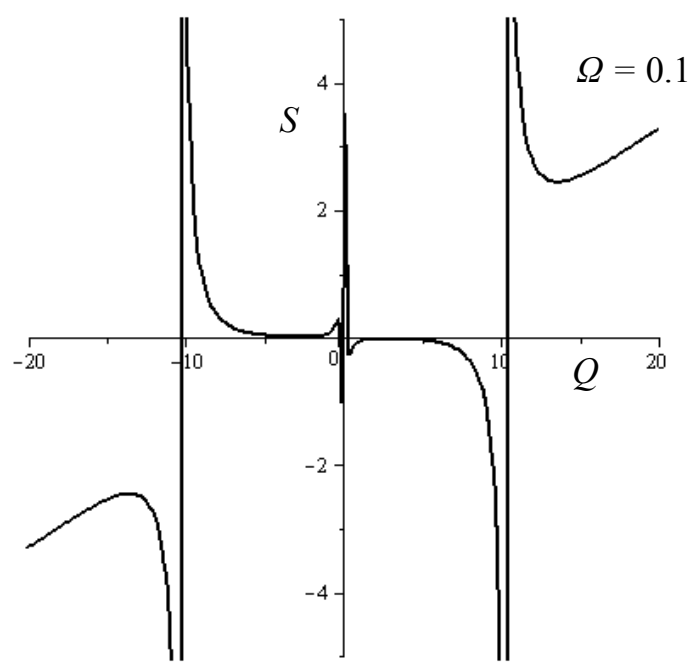

(b)

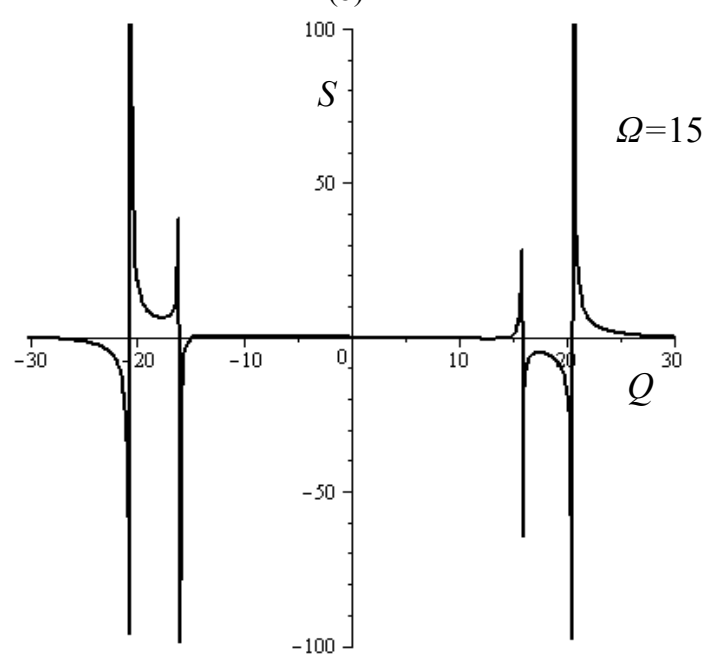

(d)

Fig. 2 The sensitivity due to guided waves $S$ is calculated as function of the wave number $Q$ for $\mu_{v}>0$ at different values of reduced frequency $\Omega=0.05,0.1,5$ and 15 . The film thickness $a=0.05 \mathrm{~cm}$. 


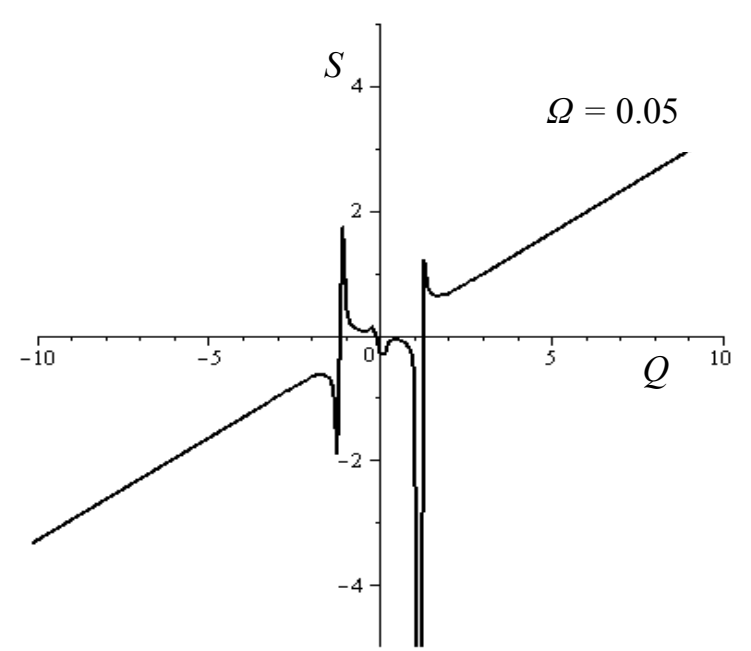

(a)

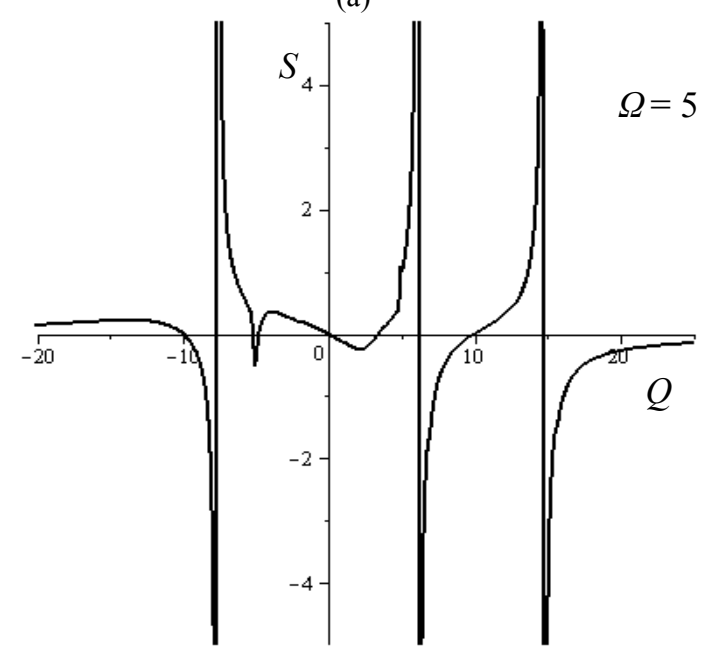

(c)

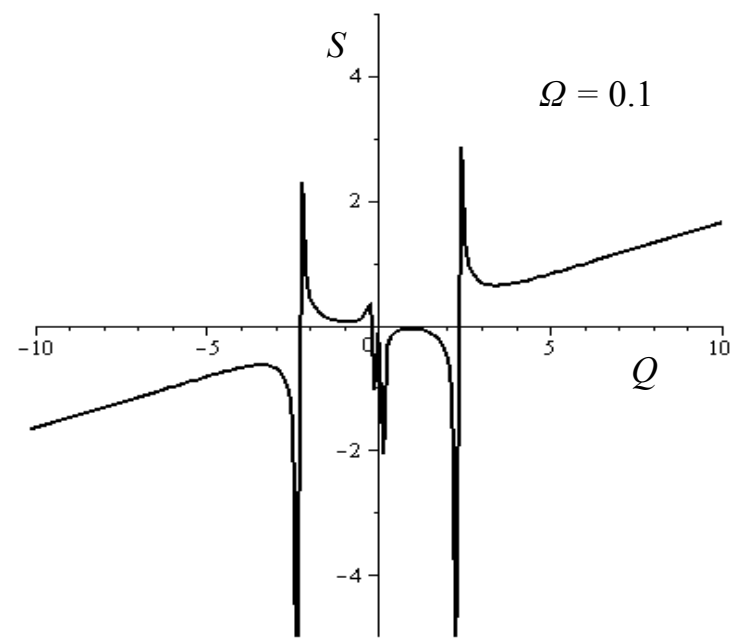

(b)

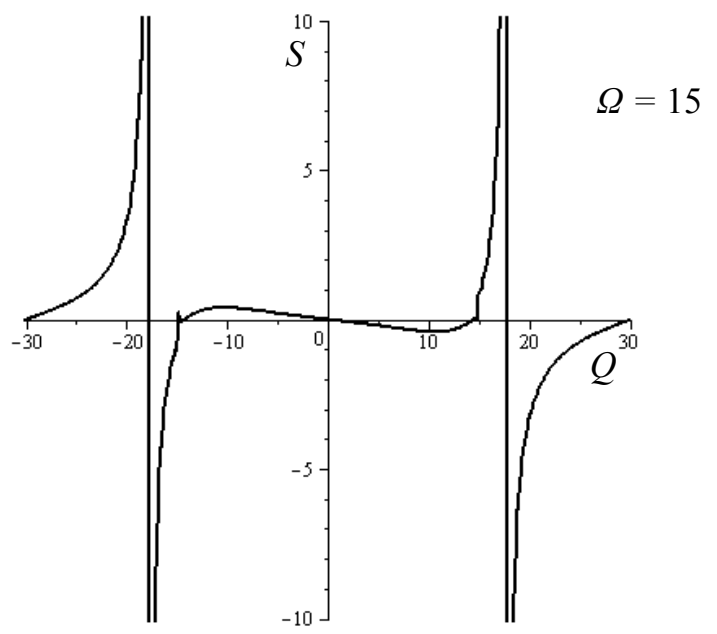

(d)

Fig. 3 The sensitivity due to guided waves $S$ is calculated as function of the wave number $Q$ for $\mu_{v}>0$ at different values of reduced frequency $\Omega=0.05,0.1,5$ and 15 . The film thickness, $a=0.15 \mathrm{~cm}$.

\subsection{Results for $\mu_{v}>0$}

In this case, the reduced frequency is tuned such that $\mu_{v}>0$. The sensitivity $S$ is plotted in Fig. 1 as function of wave number $Q$ at the chosen set of values of reduced frequencies $\Omega=0.05,0.1,5$ and 15 . The calculation has performed using the following values: $a=0.05 \mathrm{~cm}, \Omega_{m}=5, \Omega_{m} \cdot \Omega_{a}=0.1, n_{1}=1, \varepsilon_{2}=1.0, \varepsilon_{3}$ $=-1, \mu_{3}=-4, \Omega_{0}=0.3, \mu_{02}=2$.

From Fig. 2, we can see that $S$ varies as the wave number changes. The sensitivity goes to zero at the zeros of wave number $Q$ which corresponds to the zeros of $\omega$ and infinities at the poles of $\omega$ as stated in Eqs. (7) and (8). The position of the zeros and infinities changes as $\Omega$ changes. Fig. 2 gives us a promising sensor for the stated values. The system is very sensitive with changes occurs at the cladding layer. In this case, the maximum value, $S= \pm 7$, occurs at $\Omega=0.05$ and at $Q= \pm 20$.

To study the effect of the film thickness, the same calculation is repeated keeping all parameters unchanged except the film thickness value is changed to $a=0.15 \mathrm{~cm}$. The sensitivity, $S$, is plotted in Fig. 3 versus the wave number, $Q$, at $\Omega=0.05,0.1,5$ and 15 .

We notice that the maximum sensitivity, $S= \pm 3$, occurs at $\Omega=0.05$ at $Q= \pm 3$. The figure exhibits the zeros and poles which is associated with $\omega$. We see the effect of film thickness appears at the limits of the propagation constant, $Q$, which decreases dramatically 
with increasing $a$. Also, the maximum value of $S$ decreases with respect to the previous calculations.

The effect of changing MTM's parameters are done by repeating the previous calculations keeping all parameters unchanged and swamping the values of $\varepsilon_{3}$ and $\mu_{3}$; i.e., $\varepsilon_{3}=-4, \mu_{3}=-1$. Fig. 4 exhibits the sensitivity, $S$, versus the wave number, $Q$, at $a=0.05$ $\mathrm{cm}$ and $\Omega=0.05,0.1,5$ and 15 .

The maximum sensitivity $S= \pm 100$ occurs at $\Omega=$ 0.05 and $Q= \pm 10$. From previous calculation, we can control the sensitivity of the sensor by changing the value of the film thickness and the MTM's parameters.

\subsection{Results for $\mu_{v}<0$}

The reduced frequency is tuned such that $\mu_{\mathrm{v}}<0$. The sensitivity $S$ is displayed in Fig. 5 as function of wave number $Q$ at chosen set values of reduced frequencies $\Omega=3.765,3.7625,6.8$ and 6.95 that satisfied the condition $\mu_{\mathrm{v}}<0$. The calculation has performed using the following values: $a=0.05 \mathrm{~cm}$, $\Omega_{m}=5, \Omega_{m} \cdot \Omega_{a}=0.1, n_{1}=1, \varepsilon_{2}=1.0, \varepsilon_{3}=-1.0, \mu_{3}=-4$, $\Omega_{0}=0.3, \mu_{02}=2$.

The sensitivity, as presented in Fig. 5, has its maximum value, $S=7$, at $\Omega=6.7625$ and $Q=10$.

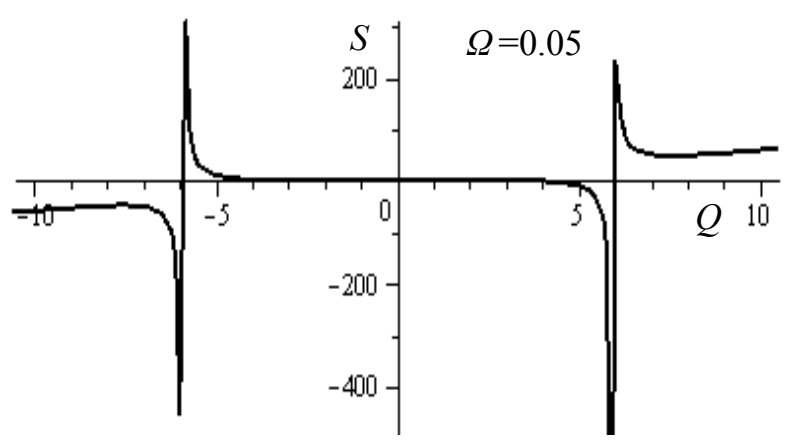

(a)

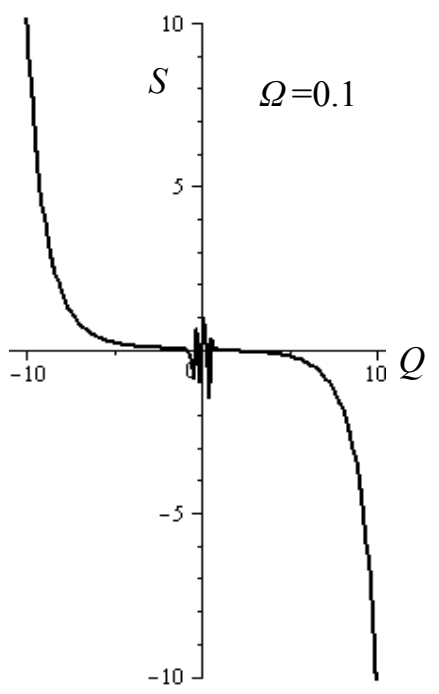

(b)

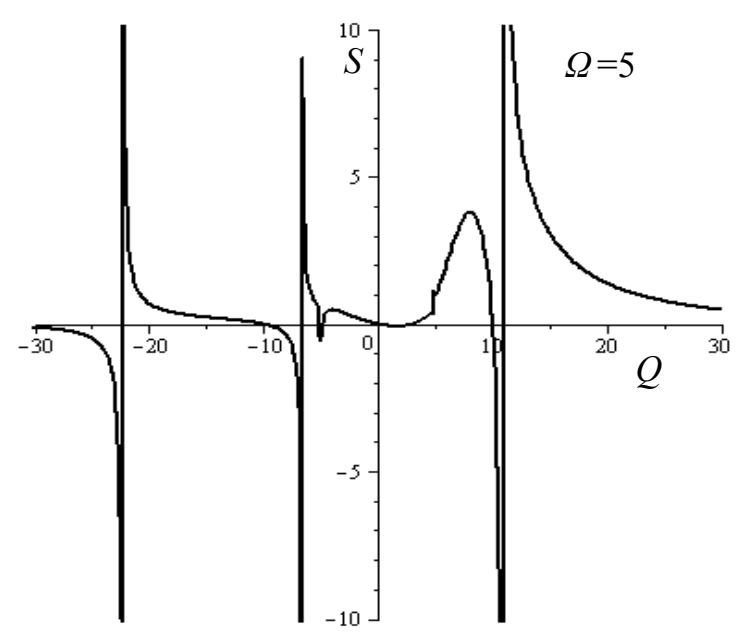

(c)

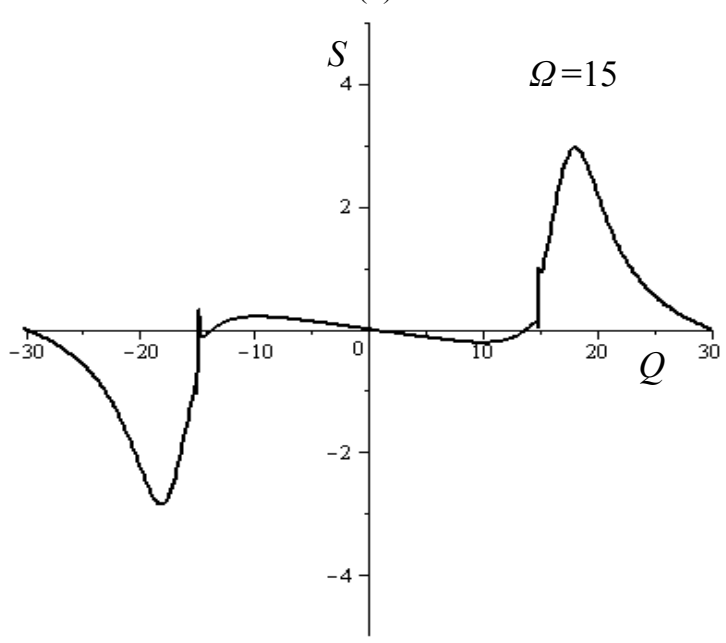

(d)

Fig. 4 The sensitivity $S$ is calculated as function of the wave number $Q$ for $\mu_{v}>0$ at different values of reduced frequency $\Omega$ $=0.05,0.1,5$ and 15 . The film thickness, $a=0.05 \mathrm{~cm}$ and $\varepsilon_{3}=-4.0, \mu_{3}=-1$. 


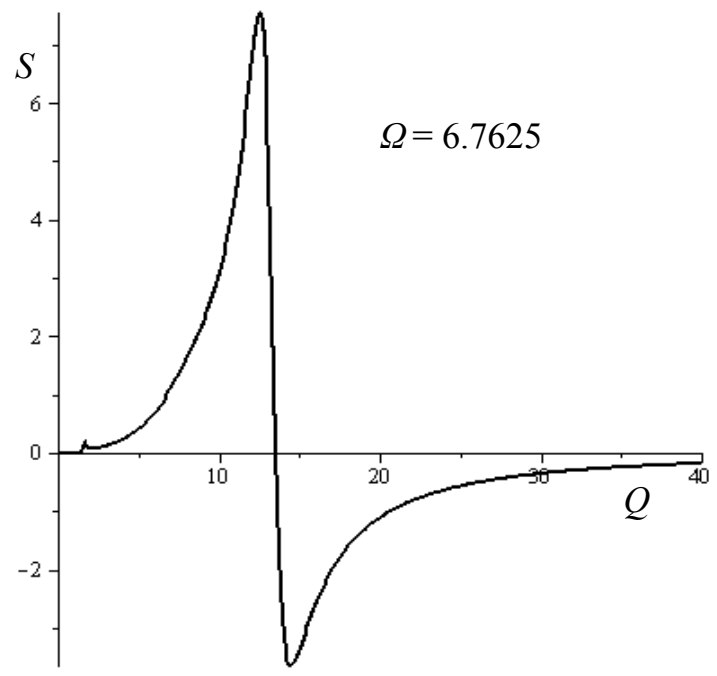

(a)

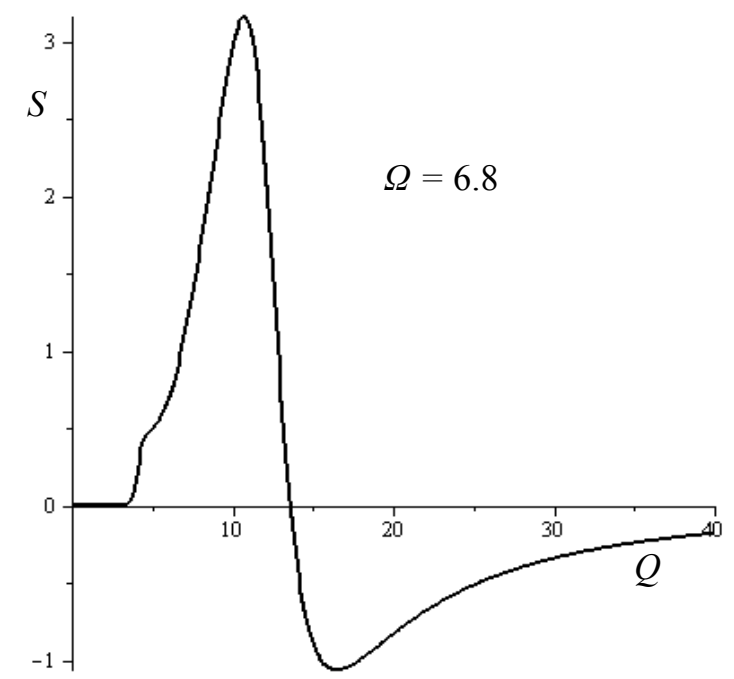

(c)

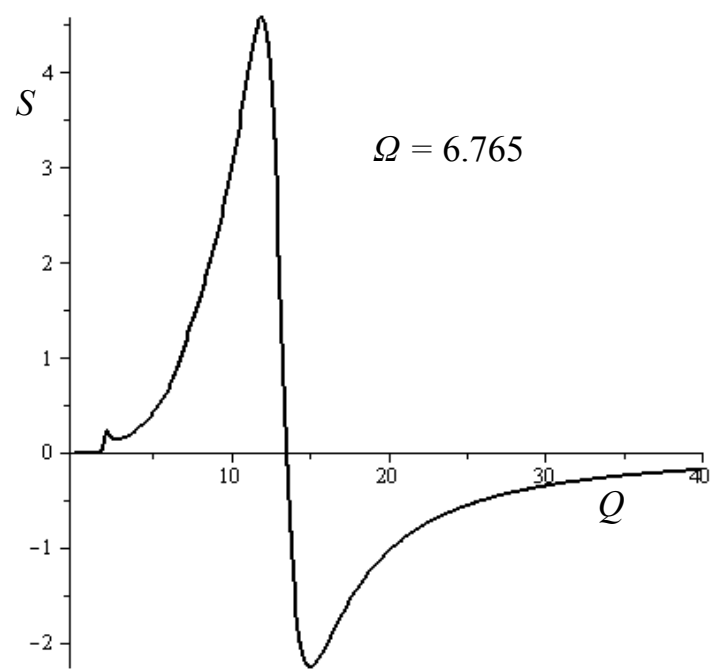

(b)

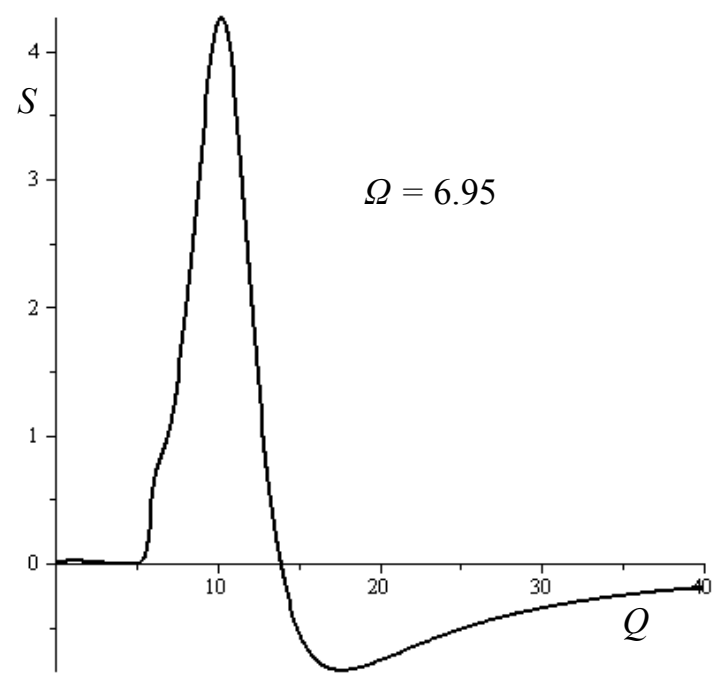

(d)

Fig. 5 The sensitivity $S$ is calculated as function of the wave number $Q$ for $\mu_{v}<0$ at different values of reduced frequency $\Omega$ $=6.7625,6.765,6.8$ and 6.95 .

\section{Conclusions}

The homogenous sensitivity of the proposed sensor is studied. The sensor is a three-layer waveguide consists of a LHM film surrounded by AF substrate and dielectric cladding. The sensitivity is calculated for range of frequencies at which the Voigt frequency is negative and positive. Moreover, we studied the effect of the film thickness and the LHM parameters. Results show that the sensitivity of the proposed sensor can be tuned by changing the value of the film thickness and the LHM's parameters. The results might be of interest to the sensors fabricators.

\section{References}

[1] Ritchie, R. H. Plasma Losses by Fast Electrons in Thin Films. Phys. Rev. 1957, 106, 874-881.

[2] Schuster, S. C.; Swanson, R. V.; Alex, L. A.; Bourret, R. B.; Simon, M. I. Assembly And Function of a Quaternary Signal Transduction Complex Monitored by Surface Plasmon Resonance. Nature (London) 1993, 365, 343-347.

[3] Gong, J.; Chu, Q. In A Novel SCLH Transmission Line Structure and Its Application to UWB Filter Design, Proceedings of International Workshop on Metamaterials, South China Univ. of Technol., 2008.

[4] He, Y.; He, P.; Harris, V. G.; Vittoria, C. Role of Ferrites in Negative Index Metamaterials. IEEE Trans. Magnetized 2006, 42, 2852-2854. 
[5] Wu, R. X. Effective Negative Refraction Index in Periodic Metal-Ferrite-Metal Film Composite. Journal of Applied Phys. 2005, 97, 076105-076108.

[6] Rachford, F. J.; Armstead, D. N.; Harris, V. G.; Vittoria, C. Simulations of Ferrite-Dielectric-Wire Composite Negative Index Materials. Phys. Rev. Lett. 2007, 99, 057202-057206.

[7] Thibaudeau, C.; Caillé, A. Interface and Guided Magnetic Polariton Modes in Magnetic Structure. Phys. Rev. B 1985, 32, 5907-5917.

[8] El-Khozondar, H. J.; Al-Sahhar, Z. I.; Shabat, M. M. Electromagnetic Surface Waves of a Ferrite Slab Bounded by Metamaterials. International Journal of Electronics and Communications (AEU) 2010, 64, 1063-1067.

[9] Al-Sahhar, Z. I.; Shabat, M. M.; El-Khozondar, H. J. Magnetostatic Surface Waves Propagation at Dissipative Ferrite-MTMs-Metal Structure. Journal of Superconductivity and Novel Magnetism 2013, 2, 584-594.

[10] El-Khozondar, R.; El-Khozondar, H.; Shabat, M. Surface Wave Propagation in Ferroelectric/MTMS Interface. Integr. Ferroelectr. 2011, 130, 50-57.

[11] Veselago, V. G. The Electrodynamics of Substances with Simultaneously Negative Values of Permittivity and Permeability. Soviet Phys. Uspekhi. 1968, 10, 509-514.

[12] Pendry, J. B.; Holden, A. J.; Robbins, D. J.; Stewart, W. J. Magnetism from Conductors and Enhanced Nonlinear Phenomena. IEEE Trans. Microwave Theory Tech. 1999, 47, 2057-2084.

[13] Pendry, J. B.; Holden, A. J.; Stewart, W. J.; Youngs, I. Extremely Low Frequency Plasmons in Metallic Mesostructures. Physical Review Letter 1996, 76, 4773-4776.

[14] Shelby, R. A.; Smith, D. R.; Schultz, S. Experimental Verification of Negative Index of Refraction. Science 2001, 292, 77-79.
[15] Pendry, J. B. Negative Refraction Makes a Perfect Lens. Physical Review Letter 2000, 85, 3966-3969.

[16] Fang, N.; Lee, H.; Sun, C.; Zhang, X. Sub-Diffraction-Limited Optical Imaging with a Silver Superlens. Science 2005, 308, 534-537.

[17] Taya, S. A.; El-Khozondar, H. J.; Shabat, M. M.; Mehjez, E. M. TM Nonlinear Waveguide Slab Optical Sensor Utilizing Left-Handed Materials. Journal of Functional Materials 2011, 18, 1-5.

[18] El-Khozondar, H. J.; Taya, S. A.; Shabat, M. M.; Mehjez, E. M. Lossy Double Negative Guiding Layer Optical Sensors. Opto-Electronics Review 2011, 19, 277-281.

[19] El-Khozondar, H.; Müller, M.; El-Khozondar, R.; Shabat, M.; Koch, A. In Temperature Sensitivity of TE Double-Negative Metamaterial Optical Sensor, Proceedings of Poc. of SPIE 7390, 2009.

[20] El-Khozondar, R. J.; El-Khozondar, H. J.; Shabat, M. M. Double-Negative Metamaterial Optical Waveguide Behavior Subjected to Stress. Islamic University Journal 2008, 16, 9-20.

[21] El-Khozondar, H. J.; El-Khozondar, R. J.; Shabat, M. M.; Koch, A. W. In Metallic Nonlinear Magnetooptical Nonreciprocal Isolator, Proceedings of SPIE Europe-Optical Metrology 2009 International Symposium, Germany, June 15-18, 2009.

[22] Brioude V.; Parriaux, O. Normalised Analysis for the Design of Evanescent-Wave Sensors and Its Use for Tolerance Evaluation. Optical and Quantum Electronics 2000, 32, 899-908.

[23] Tiefenthaler, K.; Lukosz, W. Sensitivity of Grating Couplers as Integrated-Optical Chemical Sensors. J. Opt. Soc. Amer. B. 1989, 6, 209-220.

[24] Hartstein, A.; Burstein, E.; Marasusin, A. A.; Brewer, B.; Wallis, R. F. Surface Polaritons on Semi-infinite Gyromagnetic Media. J. Phys. C. 1973, 6, 1266-1276. 